\title{
Incremental threshold loading: a standard protocol and establish- ment of a reference range in naive normal subjects
}

\author{
P.H. Johnson, A.J. Cowley, W.J.M. Kinnear
}

Incremental threshold loading: a standard protocol and establishment of a reference range in naive normal subjects. P.H. Johnson, A.J. Cowley, W.J.M. Kinnear. OERS Journals Ltd 1997.

ABSTRACT: Incremental threshold loading (ITL) has been proposed as a test of inspiratory muscle strength and endurance. To date, however, there has been no standardized protocol for an ITL test, and no reference range, with different investigators using a variety of different pressure increments in small numbers of subjects.

We developed an ITL test using the weighted plunger (WP) principle, which uses standard increments of pressure. In our protocol subjects inspire through the WP generating an initial threshold opening pressure of $10 \mathrm{cmH}_{2} \mathrm{O}$. This pressure is raised at 2 min intervals in increments of $5 \mathbf{c m H}_{2} \mathrm{O}$ until they fail to lift the plunger on two consecutive attempted breaths. Sixty healthy volunteers (30 males and 30 females) aged 20-80 yrs performed the ITL test. Twelve subjects (six females and six males) performed the test twice to assess reproducibility and repeatability. Using stepwise multiple linear regression, we regressed the maximum threshold pressure sustained for a full 2 min $(P \max )$ against age, height, weight and static maximum inspiratory mouth pressure (MIP).

$P \max$ was significantly related to age but not to either height or weight, the regression equation for males was $P_{\max }\left(\mathrm{cmH}_{2} \mathrm{O}\right)=103.8-(1.0 \times$ age in years $)$, and for females was $P \max \left(\mathrm{cmH}_{2} \mathrm{O}\right)=93.7-(1.0 \times$ age in years $)$. The within-subject standard deviation for those repeating the ITL test was $5.4 \mathrm{cmH}_{2} \mathrm{O}$.

Incremental threshold loading is a simple technique with good reproducibility, which most naive subjects can use without difficulty. By using standard pressure increments and performing the test in a large number of naive subjects, we have established a reference range that should be applicable wherever similar pressure increments are used.

Eur Respir J 1997; 10: 2868-2871.
University Hospital, Queens Medical Centre, UK.

Correspondence: W.J.M. Kinnear

University Hospital

Queens Medical Centre

Nottingham

NG7 2UH

UK

Keywords: Normal range

respiratory muscles

threshold loading

weighted plunger

Received: February 191997

Accepted after revision July 51997
Inspiratory muscle strength can be assessed by measurement of transdiaphragmatic or mouth pressure during maximal static inspiratory manoeuvres or maximal sniffs [1-3], but this does not assess inspiratory muscle endurance (IME), which more accurately reflects the normal function of respiratory muscles. Methods have been described for measuring IME using three different techniques: sustained maximal voluntary ventilation (MVV) [4], inspiratory resistive loading [5] and inspiratory threshold loading [6]. There are drawbacks associated with each of these techniques, rendering them impractical for use with large numbers of patients or healthy subjects in research projects. MVV requires cumbersome and expensive equipment, and a degree of co-operation from subjects which many patients would find difficult. Resistive loading produces results that are poorly reproducible and particularly susceptible to changes in breathing patterns [7, 8]. Parameters such as tidal volume and duty cycle must be kept constant to obtain comparable results, and this would also be beyond the capability of many patients.

Using the weighted plunger principle [6], MARTYN et al. [9] proposed an incremental threshold loading (ITL) protocol as a test of inspiratory muscle performance over time, and this was compared with maximal threshold loading by McElvaneY [10] who found that there was less intraindividual variation with the incremental method. ITL starts with a low threshold opening pressure, providing subjects with a "learning" period during the early stages during which they can accustom themselves to the test. Although there has been debate as to whether ITL provides a measure of endurance or fatigue of inspiratory muscles [11], the technique has advantages in that it is not affected by changes in breathing pattern $[9,12]$ and has been shown to be reproducible $[9,10]$. It is, therefore, the most suitable technique for development as a test that can be used on a large scale with widely differing groups of subjects.

The basic equipment for ITL is the weighted plunger first described by NicKerson and KeENS [6]. Previous in-vestigators have built similar systems and generated the pressure increments by adding standard weights (usually 100 g). As each system uses a different sized valve aperture, the pressure increments vary quite widely between laboratories. The number of subjects studied in previously published work has also been small $[9,11]$, and in many cases the subjects were highly trained in the technique. There- 
fore, we developed an ITL test using standard increments of pressure, and have established a reference range by recruiting a large number of naive volunteers to perform the test. This will enable other investigators to construct similar systems and obtain comparable results.

\section{Materials and methods}

In our workshops we constructed a weighted plunger based on the design of NiCKERSON and KeENS [6]. The valve is illustrated in figure 1. To emulate the refinement of the original design described by EASTWOOD and HILLMAN [13], our valve produces a seal using a rubber ring with a "V" shaped cross-section. We have previously established that, in our system, pressure is independent of flow over physiological ranges [14].

In our protocol, the threshold pressure was raised at 2 min intervals in increments of $5 \mathrm{cmH}_{2} \mathrm{O}$, starting with a threshold pressure of $10 \mathrm{cmH}_{2} \mathrm{O}$. Increments were generated by the addition of $135 \mathrm{~g}$ weights to the plunger. Subjects inspired through the plunger while seated, using a mouthpiece and wearing a noseclip. They were asked to breathe continuously through the apparatus until they were unable to lift the plunger on two consecutive attempted breaths. No instructions were given regarding breathing pattern or frequency. At exhaustion the maximum pressure sustained for a full $2 \mathrm{~min}\left(P_{\max }\right)$ is documented. $P \max$ was also expressed as a percentage of the subject's maximal inspiratory mouth pressure (MIP). The pressure signal from the valve outlet was passed through an analogue-digital converter (SiPlan Electronics Research, Stratford-upon-Avon, UK) to an Archimedes A310 Computer (Acorn computers, Cambridge, UK). The respiratory rate and duty cycle can be calculated from the pressure data. Statistical analysis was performed with the FASTAT program (Systat Inc. Evanston, IL, USA). Using

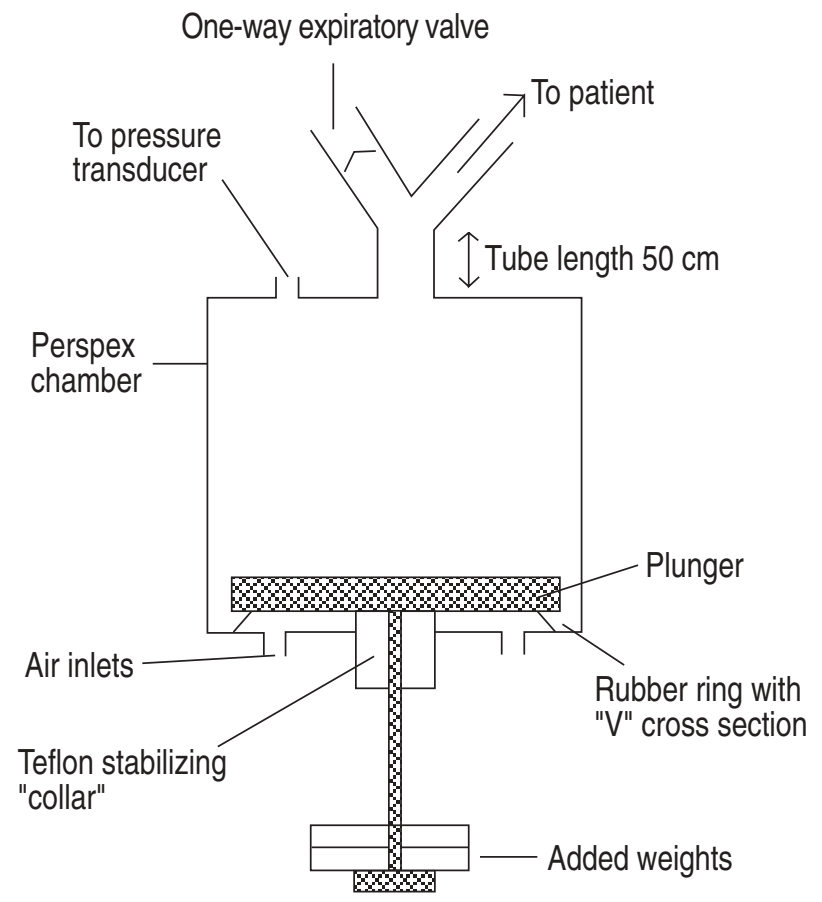

Fig. 1. - The weighted plunger. stepwise multiple linear regression, $P$ max was regressed against age, height, weight and MIP. The level of statistical significance was taken as a p-value of less than 0.05 .

Sixty healthy volunteers (30 males, 30 females) performed the ITL test. Volunteers were recruited from hospital staff, and the registration list of a local General Practitioner. We excluded any subjects with a history of disease with the potential to affect the respiratory muscles. In order to achieve an even age distribution we recruited five males and five females in each decade of life from age 2079 yrs. Forty seven were lifelong nonsmokers, five had a past history of smoking (average 20 pack years) and three were current light smokers. Smoking history was unknown in five cases. Ethical approval for the study was obtained from the University of Nottingham Medical School Ethics Committee, and all subjects gave written informed consent. Height and weight were recorded for each, and spirometry was performed using a dry bellows spirometer (Vitalograph, Buckingham, UK). Measurement of MIP was made using the portable PI,max ${ }^{\circledR}$ analyser device (P.K. Morgan, Chatham, Kent, UK). For this, subjects were seated wearing a noseclip and made repeated maximal inspiratory manoeuvres from functional residual capacity (FRC) until two maximal values were obtained within $10 \%$ of one another.

To assess repeatability and reproducibility, one male and one female subject in each decade of life (total 12 subjects) performed the ITL test on two occasions at least one week apart. The within-subject standard deviation was calculated [15].

\section{Results}

All subjects had normal spirometric values: mean (SD) percentage predicted forced expiratory volume in one second was $105 \%(15.7 \%)$ and forced vital capacity was $111 \%(14.9 \%)$. All subjects also had normal MIP values according to the reference range of BRUSCHI et al. [16] measured at FRC. $P$ max was found to be related to age, but not to either height or weight. This association remained significant after MIP was taken into account, in that the ratio of $P \max /$ MIP also declined with age. The calculated regression equations for males were:

$$
\begin{gathered}
P \max \left(\mathrm{cmH}_{2} \mathrm{O}\right)=103.8-(1.0 \times \text { age in } \mathrm{yrs}) \mathrm{RSD}=18.8 \\
P \max / \mathrm{MIP}(\%)=93.4(0.6 \times \text { age in } \mathrm{yrs}) \mathrm{RSD}=22.3
\end{gathered}
$$

where RSD is the residual standard deviation. The calculated regression equations for females were:

$$
\begin{aligned}
& P \max \left(\mathrm{cmH}_{2} \mathrm{O}\right)=93.7-(1.0 \times \text { age in yrs }) \mathrm{RSD}=13.3 \\
& P \max / \mathrm{MIP}(\%)=100.0(0.5 \times \text { age in yrs }) \mathrm{RSD}=24.9
\end{aligned}
$$

Figure 2 shows $P \max$ plotted as a function of age in males and females. The r-values were 0.67 for males (fig. 2a) and 0.78 for females (fig. 2b). Figure 3 shows $P \max /$ MIP plotted as a function of age for all subjects. The decline with age was significant, with an r-value of $0.40(\mathrm{p}<$ $0.05)$.

Mean (SD) duty cycle and respiratory rate throughout the ITL test for all 60 subjects were $0.58(0.08)$ and 16.0 (4.6) breaths $\mathrm{min}^{-1}$, respectively. There was no correlation between duty cycle or respiratory rate and $P$ max. In most 

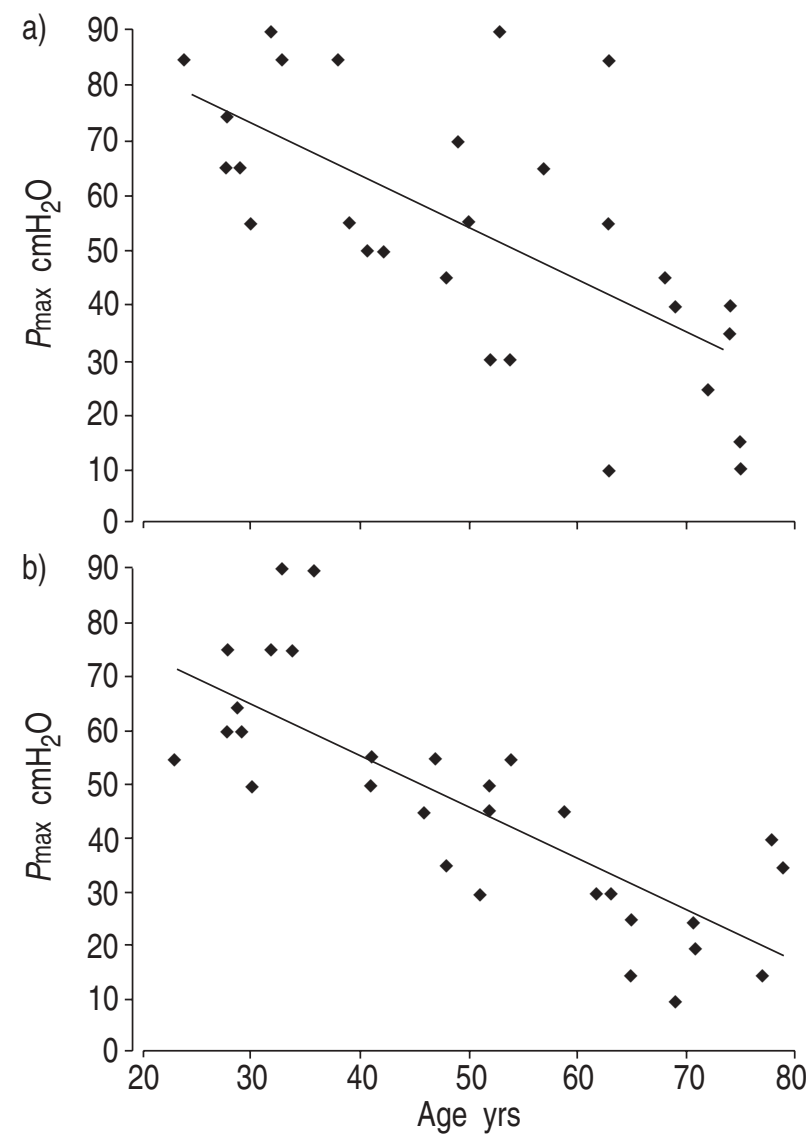

Fig. 2. - Maximum threshold pressure sustained for 2 min ( $P \max )$ plotted as a function of age in: a) males $(\mathrm{r}=0.67, \mathrm{p}<0.001)$; and $\mathrm{b})$ females $(\mathrm{r}=0.78, \mathrm{p}<0.001)$.

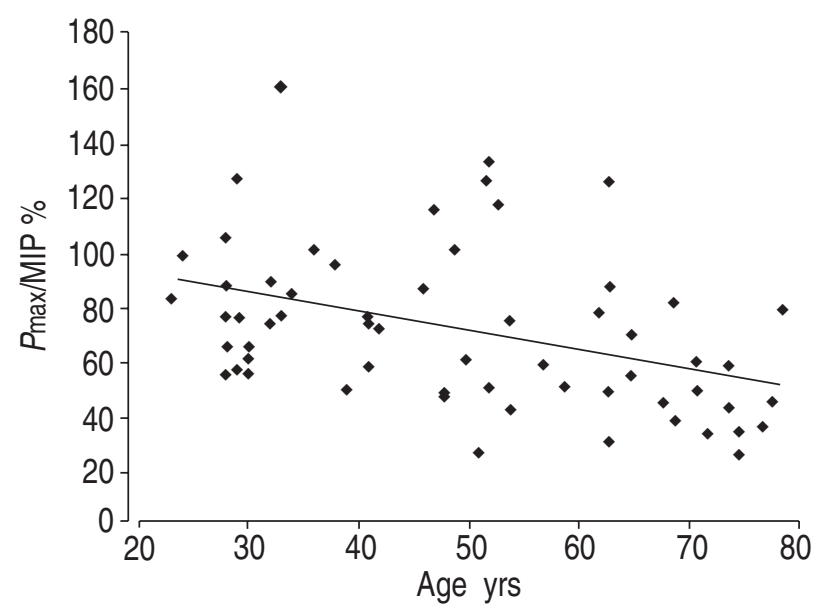

Fig. 3. - Maximum threshold pressure sustained for $2 \min \left(P_{\max }\right) / \max$ imal inspiratory pressure (MIP) plotted as a function of age for all subjects. The $\mathrm{r}$-value for the plot was $0.40(\mathrm{p}<0.05)$.

cases there was either no change or a small increase in duty cycle as ITL progressed, and respiratory rate changed very little.

For the twelve subjects repeating the ITL test, the within-subject sD for $P \max$ was $5.4 \mathrm{cmH}_{2} \mathrm{O}$.

\section{Discussion}

Although there is debate about whether ITL measures endurance or strength of inspiratory muscles [11], it pro- vides a reproducible index of the ability of inspiratory muscles to cope with increasing loads. Common pathological processes (such as chronic obstructive pulmonary disease and cardiac failure) load the respiratory muscles abnormally and ITL may provide a useful tool for investigation and research in these conditions.

The present results confirm that ITL is not susceptible to changes in breathing pattern, and we have shown for the first time that the effects of age and sex on the results obtained is important. Interestingly, in the original work of MARTYN et al. [9] an average initial duty cycle of 0.4 was reported, which fell during the ITL test. The average duty cycle throughout the ITL test in the present study was somewhat higher at 058 , and in most subjects tended to rise slightly as the test progressed. The reason for this difference from previous findings is not clear.

Previous studies of ITL have been in small numbers of subjects: MARTYN et al. [9] studied 14 subjects and EASTw00D et al. [11] only seven, most of whom were not naive. In the study of EASTWOOD et al. [11], the percentage of MIP attained at exhaustion was higher than the present study, at over $90 \%$. The subjects in question had, how-ever, been highly trained in the technique. Weiner et al. [17] found that untrained subjects reached a mean value of $69 \%$ MIP during ITL, which is very similar to the present findings. We have studied a much larger group and have shown that ITL can be performed with ease in naive subjects.

MarTYN et al. [9] and McElvANEY et al. [10] reported good repeatability for their ITL test, but gave no figures for reproducibility. We have shown that our ITL test is both repeatable (with a within-subject SD for $P \max$ of 5.4 $\mathrm{cmH}_{2} \mathrm{O}$ ) and reproducible.

Our weighted plunger system would not be difficult for other investigators to adopt, and our reference range should be applicable wherever similar pressure increments are used. No standardization of equipment would be necessary, other than to ensure that pressure is independent of airflow rate, and that the same pressure increments could be generated.

Acknowledgements: The authors wish to thank A. Merrick in the Medical Faculty Workshop of the University of Nottingham for building the weighted plunger and N. Silcock of Farndon Green Medical Centre, Nottingham, for help in re-cruiting healthy volunteers.

\section{References}

1. Black LF, Hyatt RE. Maximal respiratory pressures: normal values and relationship to age and sex. Am Rev Respir Dis 1969, 99: 696-702.

2. Miller JM, Moxham J, Green M. The maximal sniff in the assessment of diaphragm function in man. Clin Sci 1985; 69. 91-96.

3. Milic-Emili J, Mead J, Turner JM, Glauser EM. Improved technique for estimating pleural pressure from esophageal balloons. J Appl Physiol 1961; 19: 207-211.

4. Freedman S. Sustained maximum voluntary ventilation. Respir Physiol 1970; 8: 230-244.

5. Roussos C, Macklem PT. Diaphragmatic fatigue in man. J Appl Physiol 1977; 43: 189-197.

6. Nickerson B, Keens T. Measuring ventilatory muscle endurance in humans as sustainable inspiratory pressure. J Appl Physiol 1982; 52: 768-772.

7. Bellemare F, Grassino A. Effect of pressure and timing of 
contraction on human diaphragm fatigue. J Appl Physiol 1982; 53: 1190-1195.

8. McCool FD, McCann DR, Leith DE, Hoppin FG. Pressure-flow effects on endurance of inspiratory muscles. $J$ Appl Physiol 1986; 60: 299-303.

9. Martyn JB, Moreno RH, Pare PD, Pardy RL. Measurement of inspiratory muscle performance with incremental threshold loading. Am Rev Respir Dis 1987; 135: 919-923.

10. McElvaney G, Fairburn MS, Wilcox PG, Pardy RL. Comparison of two-minute incremental threshold loading and maximal loading as measures of respiratory muscle endurance. Chest 1989; 96: 557-563.

11. Eastwood PR, Hillman DR, Finucane KE. Ventilatory responses to inspiratory threshold loading and role of muscle fatigue in task failure. $J$ Appl Physiol 1994; 76 : 185-195.

12. Morrison NJ, Fairbarn MS, Pardy RL. The effect of breathing frequency on inspiratory muscle endurance during incremental threshold loading. Chest 1989; 96 : $85-88$.

13. Eastwood PR, Hillman DR. A threshold loading device for testing of inspiratory muscle performance. Eur Respir $J$ 1995; 8: 463-466.

14. Johnson PH, Cowley AJ, Kinnear WJM. Evaluation of the Threshold ${ }^{\circledR}$ trainer for inspiratory muscle endurance training: comparison with the weighted plunger method. Eur Respir J 1996; 9: 2681-2684.

15. Bland JM, Altman DG. Measurement error. BMJ 1996; 313: 744

16. Bruschi C, Cerveri I, Zoia MC, et al. Reference values of maximal respiratory mouth pressures: a population-based study. Am Rev Respir Dis 1992; 146: 790-793.

17. Weiner P, Suo J, Fernandez E, Cherniack RM. Efficiency of the respiratory muscles in healthy individuals. Am Rev Respir Dis 1989; 140: 392-396. 